\title{
The Relationship Between Intimate Partner Violence and PTSD: An Application of Cox Regression With Time-Varying Covariates
}

\author{
Mieko Yoshihama ${ }^{1,3}$ and Julie Horrocks ${ }^{2}$
}

\begin{abstract}
This study uses Cox regression with time-varying covariates to examine the relationship between intimate partner violence and posttraumatic stress disorder (PTSD) in a random sample of Japanese American women and immigrant women from Japan $(N=211)$. Because applications of survival analysis in trauma research are scarce, this paper presents the utility of this analytical approach by contrasting it with other common methods of analysis (chi-square tests and Cox regression with covariates that do not change over time).
\end{abstract}

KEY WORDS: intimate partner violence; PTSD; cumulative incidence; Cox regression.

\section{Introduction}

In this paper, we applied Cox regression with timevarying covariates to investigate the relationship between intimate partner violence and posttraumatic stress disorder (PTSD) in a random sample of Japanese American women and immigrant women from Japan. Because applications of survival analysis in trauma research (intimate partner violence research in particular) are scarce, we compared this method to other methods commonly used in studies of intimate partner violence and PTSD.

\section{Intimate Partner Violence and PTSD}

Although many studies have investigated PTSD in women who have experienced intimate partner violence, few have assessed PTSD over the course of women's lives

\footnotetext{
'School of Social Work, University of Michigan, Ann Arbor, Michigan.

${ }^{2}$ Department of Mathematics and Statistics, University of Guelph, Guelph, Ontario.

${ }^{3}$ To whom correspondence should be addressed at School of Social Work. University of Michigan, 1080 S. University, Ann Arbor, Michigan 48109-1106; e-mail: miekoy@umich.edu.
}

(Cascardi, O'Leary, Lawrence, \& Schlee, 1995; Gleason, 1993; Tolman \& Rosen, 2001). These studies found that $40-54 \%$ of battered women developed PTSD sometime in their lives. The lifetime prevalence of PTSD among battered women is much higher than the 10-14\% found in the general population of women (Breslau \& Davis, 1992; Breslau, Davis, Peterson, \& Schultz, 1997; Kessler, Sonnega, Bromet, Hughes, \& Nelson, 1995; Resnick, Kilpatrick, Dansky, Saunders, \& Best, 1993). Most studies of PTSD in battered women have used small, nonrepresentative samples, typically self-identified battered women recruited through advertisements or from assistance programs, such as battered women's shelters and counseling programs. Many studies lacked a comparison group of nonabused women and investigated samples of almost exclusively White women; Tolman and Rosen's study is a notable exception (Tolman \& Rosen, 2001). Studies of PTSD among people of Asian and Pacific Islander descent are particularly scarce and the few that exist have focused on nonintimate partner violence trauma, such as war-related atrocity (Abueg \& Chun, 1996), combatrelated trauma (Loo, 1994), and exposure to civil unrest (Kim-Hog, Chong, Blake, \& Hiley-Young, 1995). Only one study examined the relationship between intimate 
partner violence and posttraumatic stress symptoms among women of Japanese descent in the United States (Yoshihama \& Horrocks, 2002). This study found that women who had experienced injuries and/or fear for their lives, in addition to partners' emotional and physical violence, had significantly higher numbers of posttraumatic stress symptoms than did those who had not experienced partner violence.

In addition to these sampling issues, several methodological issues arise in research on intimate partner violence and PTSD. Many studies have failed to establish the temporal sequence of intimate partner violence episodes and the onset of PTSD, which makes it difficult to separate preexisting conditions from the effects of violence. Moreover, studies have typically estimated the lifetime prevalence of PTSD by the sample proportion, that is, by dividing the number of respondents with PTSD by the total number of respondents. Because some women who are free of PTSD at the time of assessment may develop PTSD later, the sample proportion is likely an underestimate of the true lifetime prevalence (to be discussed in detail below). Typically, chi-square analysis has been used to examine whether intimate partner violence is associated with an increased likelihood of lifetime PTSD (Cascardi et al., 1995; Gleason, 1993; Tolman \& Rosen, 2001). This approach also fails to account for the possibility that some observations in the sample are censored; that is, some women who have not developed PTSD at the time of the interview may develop it later in life. Moreover, this method fails to recognize that intimate partner violence is an event that occurs at a specific moment during adult life, rather than an attribute that remains constant over the lifecourse. These methodological problems limit the ability to make inferences about the relationship between intimate partner violence and PTSD.

This study addresses these issues through the use of a unique data set and a specialized statistical technique. The data set consists of information collected from a community-based random sample of Japanese American and immigrant Japanese women and includes information on the timing of intimate partner violence and the onset of PTSD. The sample included a natural comparison group-a subset of women who had never experienced intimate partner violence. The information on the timing of intimate partner violence and the onset of PTSD helped establish whether PTSD developed after the occurrence of trauma. To exploit the unique features of this data set, we used a special statistical technique from survival analysis, Cox regression with time-varying covariates. This method takes into account censoring, and the temporal sequence of the occurrence of intimate partner violence and PTSD onset (see below).

\section{Survival Analysis and Cox Regression With Time-Varying Covariates}

In survival analysis, the outcome variable is the time to some event. The task of a survival analysis is to estimate the distribution of this variable, and examine how this distribution is affected by independent variables. In this study, the event of interest is time to onset of PTSD, measured in years since birth (i.e., age). Survival analysis provides an estimate of the proportion of women who develop PTSD at a particular age, in other words, an agespecific estimate of the incidence of PTSD.

One way to visualize the changing incidence of an event is to plot the cumulative incidence function (CIF). The value of the CIF at age $t$ represents the probability that the event of interest occurred at or before age $t$. In contrast, most survival analysis software presents the survivor function (also referred to as the survival function), which provides the probability that the event has not occurred by age $t$. For this study, the event of interest is the onset of PTSD. Because we are interested in examining the risk of developing PTSD, as opposed to remaining PTSD-free, we present the CIF rather than the survivor function. The CIF has been used in previous studies of PTSD (Chilcoat \& Breslau, 1998) and intimate partner violence (Yoshihama \& Gillespie, 2002).

Another way of visualizing changing PTSD incidence is through the hazard function. In our context, the hazard can be thought of as the age-specific risk of developing PTSD. More precisely, the hazard function at age $t$ gives the instantaneous risk of developing PTSD at age $t$, given that the individual is free of the disorder (i.e., still at risk) up to that time. The hazard function is particularly informative because it considers both the changing rate of events over time, and the changing number of individuals at risk. Although the CIF necessarily increases over time, the hazard function is not subject to any such constraint. The hazard function illustrates changes in risk over the lifespan, which can help identify age groups that are at high risk for developing PTSD, to whom prevention and intervention can be targeted.

As indicated previously, some women in the sample had not experienced PTSD by the time of interview; these women are referred to as being censored. Although some of these women may develop PTSD later in life, this event will not be observed by the researcher. As far as the researcher is concerned, a woman who is censored at age 20 is no longer "at risk" of being observed to develop PTSD. Censoring is a common feature of timeto-event data, which, if ignored, can lead to substantial biases in the analysis. The techniques of survival analysis correctly take censoring into account. For instance, 
the Kaplan-Meier method builds an estimate of the CIF as a product of conditional probabilities or proportions (Kaplan \& Meier, 1958). The denominators of these proportions change according to the changing numbers of women at risk in each age group (see Allison, 1995, for more details).

The techniques of survival analysis include several parametric regression models (e.g., exponential, Weibull, log-logistic, log-normal) as well as a semiparametric model (Cox regression) that are useful in examining the effects of covariates on the distribution of the time to an event. Of these, the Cox regression model (also known as a proportional hazards model) is the most widely used. Unlike parametric regression models that assume a particular distribution for the outcome variable, Cox regression requires only that the effect of a time-fixed covariate on the hazard function be proportional. Coefficients that quantify the effect of the covariates on the hazard of PTSD can be estimated, and exponentiation of coefficients allows us to describe the effect as a hazard ratio or (roughly) a relative risk. A hazard ratio of 1 indicates that the covariate has no effect on time to PTSD onset.

Cox regression can include covariates that change over time (Allison, 1995). This is particularly salient in investigating the effects of intimate partner violence on the development of PTSD, because intimate partner violence can occur any time in a woman's life. Cox regression with only time-fixed covariates would classify women into ever-abused and never-abused groups and estimate the relative hazard or age-specific risk of PTSD onset in each group. This approach, however, does not take into account that a woman's abuse status may change over the course of her lifespan. For instance, consider a woman who experienced violence by her partner for the first time at age 35 , and developed PTSD at age 40. In Cox regression with a time-fixed covariate, she would be classified into the abused group, and her data would contribute to the estimation of the hazard of developing PTSD for the abused group at all ages. In fact, though, she is only a member of the abused group after age 35 . Cox regression with a time-varying covariate allows this woman to contribute to the nonabused group until age 35 , and thereafter to the abused group.

In the present study, we illustrate the consequence of not accounting for censoring and/or the time-varying nature of intimate partner violence by considering a very simple model with intimate partner violence as the only independent variable, and contrasting the results obtained using three different methods: chi-square analysis and Cox regression with time-fixed and time-varying covariates. Finally, a more complete model is presented, which examines the effect of intimate partner violence and other covariates on the development of PTSD over the course of women's lives.

\section{Method}

\section{Participants}

This study used a community-based random sample of 211 women of Japanese descent born in the United States or Japan, between 18 and 49 years of age, and who had had an intimate heterosexual relationship. The upper age limit was intended to minimize the effect of hormonal changes around menopause, which may affect the experience of posttraumatic symptoms. We used a list of households containing persons with a Japanese surname in Los Angeles County compiled by a survey sampling company. This method of sampling households with Japanese surnames was used in the absence of a list that enumerated all women of Japanese descent. Although this method excluded women whose surname had been changed due to interethnic or interracial marriage, we found that approximately one fourth of the respondents had a partner who was not of Japanese descent.

Following the introductory letter sent to the households, a screening telephone call was made to identify eligible women. One woman per household was selected using a random procedure. Of the 407 households determined to have at least one eligible woman, 211 women completed the interview, resulting in a response rate of $52 \%$. This rate is comparable to other studies of women's victimization that used similar in-depth face-to-face interviews (Russell \& Howell, 1983; Wyatt, 1985) or telephone interviews (Smith, 1987). The respondents did not differ from those who declined to participate with respect to marital status, region of residence, or primary language spoken; however, those women who declined to participate tended to be older than the respondents.

The mean age of the respondents was 37.2 ( $S D=$ $10.2)$. The majority $(57 \%)$ were married at the time of the interview, $10 \%$ had been separated or divorced, and $33 \%$ had never been married. Over one fourth $(27 \%)$ were born in Japan. On average, the respondents completed 15.3 years of schooling $(S D=2.0)$, and $51 \%$ were college graduates. At the time of the interview, the majority $(78 \%)$ were employed. The respondents' annual household incomes were relatively high; $36.8 \%$ reported a household income higher than $\$ 60,000$. Nevertheless, $44.8 \%$ reported a personal income of less than $\$ 15,000$ annually. The respondents were sociodemographically comparable to female residents of Japanese descent aged 18-49 in Los Angeles County based on the 1990 Census with respect 
to educational level, marital status, employment status, occupation, birthplace, and English proficiency; however, women with higher personal and household incomes were overrepresented in this study (see Yoshihama, 1999, for details). Women who reported intimate partner violence did not differ from those who did not with respect to age, educational level, employment status, relationship status, income, or country of birth.

\section{Procedure}

Written consent was obtained at the beginning of the interview. In addition to approval from the Institutional Review Board, a Certificate of Confidentiality was obtained from the National Institute of Mental Health, Department of Health and Human Services. Trained female interviewers conducted face-to-face interviews in the respondent's preferred language (English or Japanese); 46 women $(22 \%)$ chose to be interviewed in Japanese. The interview was audiotaped if the respondent consented $(67 \%)$. On average, the interview lasted $90 \mathrm{~min}$. At the conclusion of the interview, the respondent received $\$ 20$ and a list of available assistance programs, including those with services provided in Japanese. The respondents were asked whether they wished to speak to a counselor, and a referral for counseling was made if requested.

\section{Measures}

Both English and Japanese versions of a questionnaire were developed for this study. Existing standardized measures (e.g., PTSD measure) were translated from English to Japanese using a back-translation method, and other measures (e.g., intimate partner violence measure) were constructed using a decentering method (Marin \& Marin, 1991), which involved developing a questionnaire in two languages simultaneously, followed by a series of translation and back-translation between the two versions as revisions were made to either language version. Both versions of the questionnaire underwent item-by-item review by practitioners, researchers, and graduate students in social work and were pilot-tested with a convenience sample of 21 women of Japanese descent in Los Angeles County prior to the study.

\section{PTSD}

PTSD was measured by a modified form of the National Women's Study PTSD Module (Kilpatrick, Resnick, Saunders, \& Best, 1989), a measure developed specifically to examine women's responses to victimization. It assesses the symptoms of posttraumatic stress experienced during the respondent's lifetime up to the time of the interview, unlike most other measures that focus on current or past-year symptoms. Specifically, the $\mathrm{Na}$ tional Women's Study PTSD Module assesses the respondent's experiences of 17 symptoms in three domains that correspond to the DSM-IV diagnostic criteria (Diagnostic and statistical manual of mental disorders, 4th ed., American Psychiatric Association, 1994): reexperiencing the trauma, avoidance and numbing of responses, and increased arousal. The internal consistency (Cronbach's $\alpha$ ) for each of the three domains was $.66, .72$, and .64 , respectively; internal consistency was comparable between the English and Japanese versions. The respondent's PTSD status was assessed using the $D S M-I V$ criteria: at least one of five reexperiencing symptoms, three of seven avoidance symptoms, and two of five arousal symptoms. The original National Women's Study PTSD Module examines the age at which the respondent experienced specific symptoms for the first time and the timing of the last episode. In the present investigation, a modification was made; namely, the age of the respondent at onset and every subsequent recurrence of specific posttraumatic stress symptoms was obtained. A respondent must experience a certain number of symptoms in each domain simultaneously to meet the diagnostic criteria for PTSD. Thus, extra information on the timing was collected in order to determine what symptoms occurred simultaneously, which in turn allowed the assessment of the age(s) at which the DSM-IV diagnostic criteria were met. If the respondent met the diagnostic criteria multiple times, the age of PTSD onset was obtained by calculating the minimum age at which she met the criteria.

\section{Intimate Partner Violence}

Using behavior-specific questions, the respondents were asked whether they had experienced 31 types of physical violence and 3 types of sexual assault at the hands of current or former partners during their lifetimes to date. Physical violence ranged from pushing to the use of lethal weapons and included all nine items of the Physical Aggression subscale of the Conflict Tactics Scale (CTS; Straus, 1990b) with the following modifications: (1) original CTS items that contained different acts of violence (e.g., kicking, biting, or hitting with a fist) were separated into individual items, and (2) attempted and completed acts of violence were also differentiated. The CTS items were augmented by items drawn from other studies and professional consultations (see Yoshihama, 1999, for 
specific types of violence examined). Three sexual assault items were adopted from the Sexual Experience Survey (Koss, Gidycz, \& Wisniewski, 1987) and assessed whether the respondent was forced to have sex: (1) when under the influence of alcohol or drugs or when her judgment was otherwise impaired; (2) with verbal coercion; and (3) with physical coercion. Based on this information, we created a dichotomous variable that recorded whether the respondent had ever experienced physical or sexual intimate partner violence. The minimum age at which the respondent first experienced intimate partner violence was calculated. We combined physical and sexual violence because partners' sexual assault is strongly associated with physical violence and the age of the first physical and sexual violence incidents were highly correlated.

\section{Childhood Abuse}

The respondent was asked whether she had been abused as a child, and if so, whether the abuse was physical or sexual in nature. Only two respondents reported having experienced childhood sexual abuse, and both of these women also reported childhood physical abuse. Therefore, we created a dichotomous variable representing the experience of childhood abuse.

\section{Sexual Victimization by Nonintimates}

The respondent reported whether she had experienced: (a) stalking, (b) unwanted touching of breasts and/ or genital areas, and (c) sexual assault at the hands of someone other than an intimate partner, and if so, the age at which she experienced each type of victimization for the first time. The smallest value was used as the age of the first sexual victimization by nonintimates.

\section{Immigration Status}

Each respondent was asked whether she was born in the United States or Japan, and those born in Japan were asked at what age they relocated to the United States. Instead of the respondent's country of birth, we dichotomized the respondents into 1st generation (those who immigrated to the United States at age 13 or older, $n=44$ ) and $1.5+$ generation (those who immigrated to the United States before age 13 or were born in the United States, $n=167$ ). This classification was used because those who immigrated to the United States during their preteen years or earlier (sometimes referred to as the 1.5 generation) are likely to have different life experiences than those who immigrated as adolescents; the life experiences of the 1.5 generation may be similar to the ex- perience of those who were born in the United States (Allensworth, 1997; Myers \& Cranford, 1998).

\section{Statistical Analysis}

All statistical analyses were performed in SAS, version 8.0. We used methodologies from survival analysis (e.g., Kaplan-Meier, Cox regression) to model the time to first occurrence of PTSD, with age of the respondent as the time scale. We used the Kaplan-Meier method in SAS Proc Lifetest to estimate the survivor functions used to construct the CIF plot. The value of the CIF at time $t$ was calculated as 1 minus the survivor function at time $t$. Chi-square tests and Cox regression (SAS Proc PHREG) with time-fixed and time-varying covariates were used to assess the effect of intimate partner violence on PTSD. We chose to use Cox regression rather than a parametric model because the commonly available parametric models do not accommodate the U-shaped hazard function seen in this data (see Fig. 2). Time of PTSD onset was recorded as the age (in years) at which a woman first experienced PTSD. The discrete nature of this measurement resulted in 21 tied PTSD times. We used the "ties = exact" option in SAS Proc PHREG, which is appropriate because we believe that these tied events actually occurred at different times, but the exact ordering of the event times is unknown (Allison, 1995). For the model with a time-fixed covariate, the proportional hazards assumption was checked graphically. To estimate the hazard function, we used the baseline statement and counting process style of input in SAS Proc PHREG, as outlined in Therneau and Grambsch (2000). Smooth plots of the hazard function were made using a SAS macro written by Allison.

\section{Results}

\section{Experiences of Intimate Partner Violence and Probability of Developing PTSD}

Of the 211 respondents, $115(55 \%, 95 \% \mathrm{Cl}=0.48$, $0.61)$ reported having experienced intimate partner violence, and $30(14 \%, 95 \% \mathrm{CI}=0.10,0.19)$ had experienced PTSD sometime in the past. Figure 1 shows cumulative incidence functions (CIF) for PTSD (solid line) and intimate partner violence (dashed line). As noted above, the value of the CIF at a given age provides an estimate of the probability of experiencing the event at or before that age. The estimated probability of having experienced intimate partner violence by age 25 , for example, is $.36(95 \% \mathrm{CI}=0.30,0.43)$, whereas the estimated probability of having experienced PTSD by age 25 is .08 (95\% 


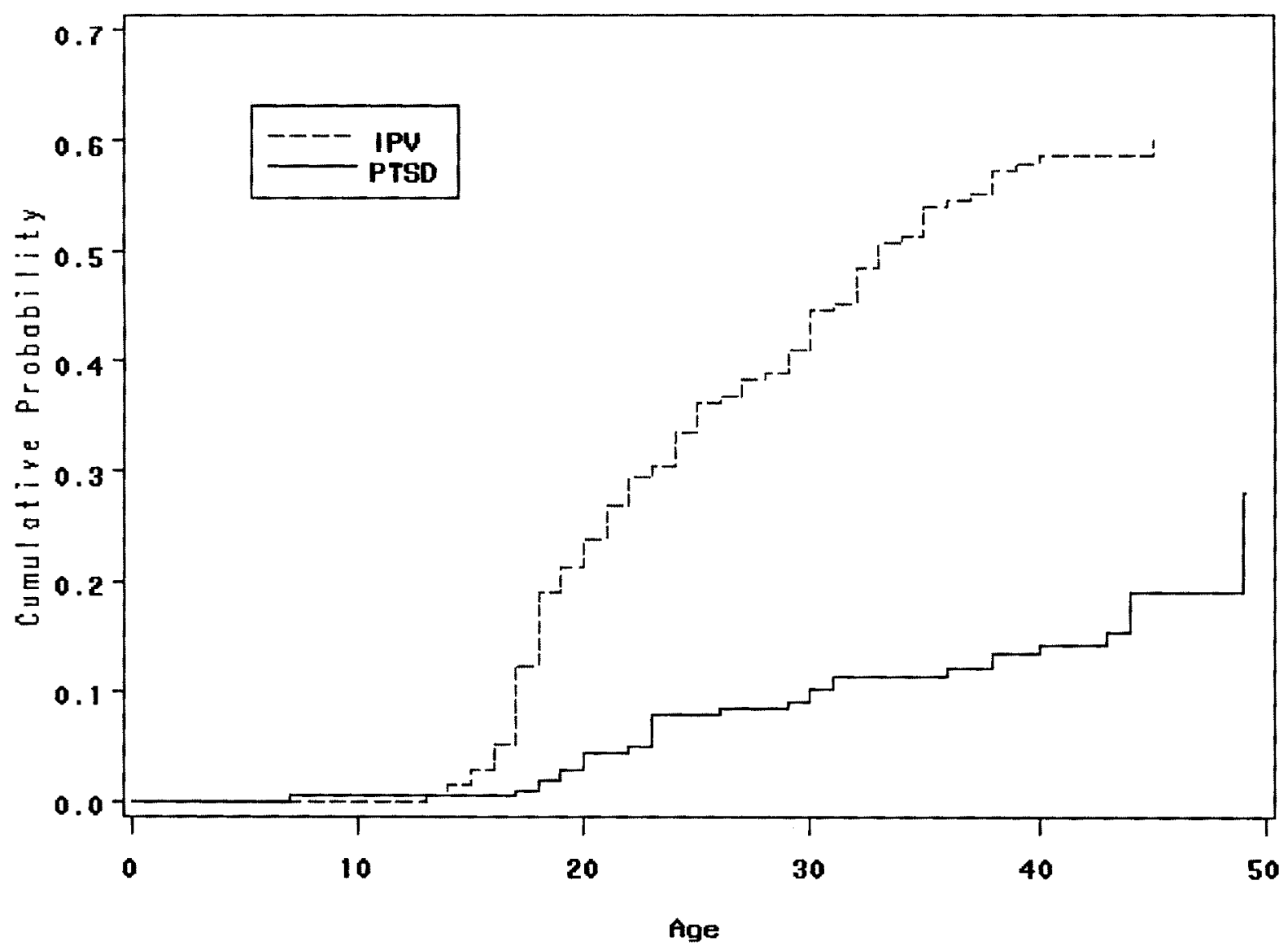

Fig. 1. Cumulative incidence functions showing the cumulative probability of experiencing intimate partner violence (IPV) and posttraumatic stress disorder (PTSD) by age of respondent.

$\mathrm{CI}=0.04,0.12)$. The proportion of women who develop PTSD is much lower than the proportion who experience intimate partner violence. The largest increase in PTSD onset appears to occur in the mid-20s and during the 40s, as evidenced by the steep slope of the CIF during this period. In contrast the greatest increase in intimate partner violence occurs during the late teen years.

The Kaplan-Meier estimate of the probability of developing PTSD by age 49 (the maximum age of the respondents) is $.28(95 \% \mathrm{CI}=0.10,0.46)$. In contrast, the usual sample proportion estimate of lifetime PTSD is $30 / 211$ or $14 \%$, which is much lower.

\section{Effects of Intimate Partner Violence on the Development of PTSD}

For illustrative purposes, we investigated the effects of intimate partner violence on the development of PTSD and contrasted the results obtained using three different methods. The first method of analysis-the one most commonly used in research on intimate partner violence-is a chi-square test of the independence of intimate partner violence and PTSD. Of the 115 respondents who experienced intimate partner violence, $21(18 \%)$ developed PTSD, as compared with $9(9 \%)$ of the 96 respondents who never experienced intimate partner violence. This gave a risk ratio or relative risk of $0.18 / 0.09=2$. A chi-square test of independence was marginally significant, $\chi^{2}(1, N=211)=3.39, p=.07 .{ }^{4}$ This test can be faulted on two fronts. First, it does not take into account censoring. Second, it ignores the fact that intimate partner violence occurs in the midst of adult life, rather than being present from birth.

\footnotetext{
${ }^{4} \mathrm{~A}$ likelihood ratio test from logistic regression, with a single dichotomous covariate indicating lifetime intimate partner violence experience, is statistically equivalent to the chi-square test discussed here. The advantage of logistic regression is that other covariates can be controlled in the analysis.
} 
The second method of analysis is Cox regression with a time-fixed covariate, a dichotomous covariate that indicates whether a respondent ever experienced intimate partner violence. The estimated hazard ratio is 2.11 (95\% CI = $0.97,4.61)$. Thus, the hazard or risk of developing PTSD was 2.11 times higher for those who experienced intimate partner violence than for those who never experienced intimate partner violence, which was marginally significant $(p=.06)$. Although this analysis does take into account the fact the some women are censored, it does not account for the fact that intimate partner violence status can change in midlife.

For the third method, Cox regression with a timevarying covariate, we created a variable that takes the value zero up until the time a woman experienced intimate partner violence, and the value one thereafter, using special syntax in SAS Proc PHREG (see Allison, 1995, for details). Using this method, the hazard of PTSD onset was estimated to be 3.34 times higher after experiencing intimate partner violence $(95 \% \mathrm{CI}=1.54,7.26)$, which was highly significant $(p<.01)$. A visualization of the effect of this time-varying covariate is given in Fig. 2.
For a woman who experienced intimate partner violence at age 25, the instantaneous risk (or hazard) of developing PTSD jumps at age 25 from approximately 0.0045 to over three times that number, namely 0.015 . Note that the magnitude of the hazard is very small, as it represents an instantaneous rate of change.

We investigated the duration of the effect of intimate partner violence by including an interaction between the time-varying covariate indicating intimate partner violence and time (Allison, 1995). If the effect of intimate partner violence decreases linearly over time, we would expect the hazard ratio to be less than 1 . Our results indicated a nonsignificant interaction in the expected direction (hazard ratio $=0.95, p=.16$ ).

Next we added the respondent's experience of sexual victimization by nonintimates as a time-varying covariate in the model, as well as childhood abuse and immigration status, which remain constant over the adult lifespan. Although income, employment, education, and marital status at the time of interview were recorded, these variables were not included in the model. These conditions change over the adult lifespan and may be the

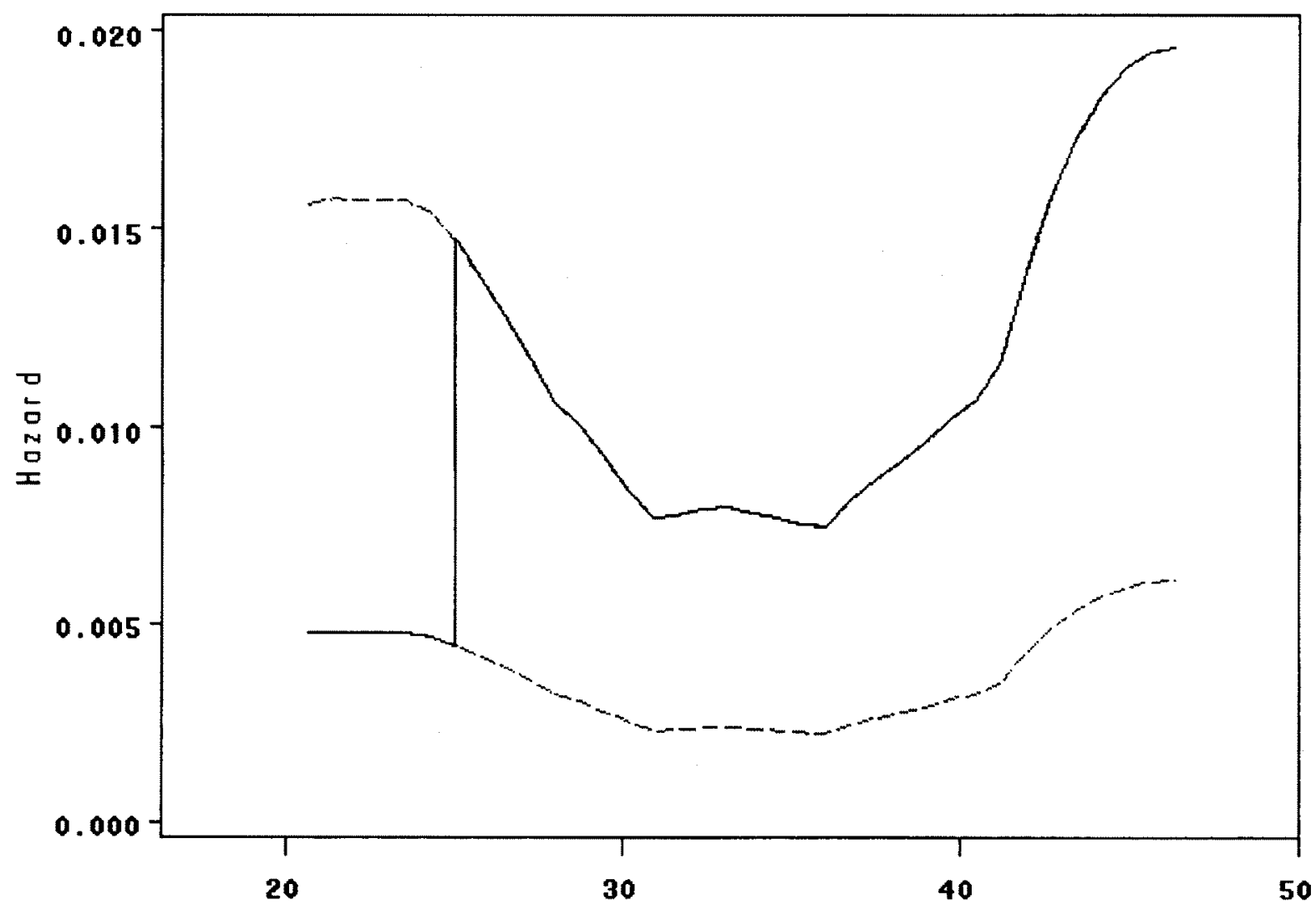

Fig. 2. Hazard plot showing risk of developing PTSD for a woman who experiences intimate partner violence at age 25. Upon experiencing intimate partner violence, her risk of PTSD jumps from the lower line to the upper line. 
Table 1. Results of Cox Regression Predicting the Likelihood of Developing PTSD in Japanese American Women

\begin{tabular}{lcccc}
\hline & $\beta$ & $S E$ & Hazard ratio & $95 \% \mathrm{CI}$ \\
\hline Intimate partner violence & 1.08 & 0.41 & $2.94^{* *}$ & $1.32,6.54$ \\
Sexual victimization & 0.70 & 0.39 & $2.00^{+}$ & $0.93,4.31$ \\
$\quad$ by nonintimates & & & & \\
Childhood abuse & 0.64 & 0.41 & 1.90 & $0.65,4.22$ \\
Immigration status $^{a}$ & 0.05 & 0.43 & 1.06 & $0.46,2.44$ \\
\hline
\end{tabular}

Note. $N=211,30$ events and 181 censored cases; likelihood ratio

$\chi^{2}(4, N=211)=15.71, p<.01$.

" First generation as reference group.

${ }^{+} p<.10{ }^{* *} p<.01$

result of, rather than contributing factors for, partner abuse. As shown in Table 1, controlling for sexual victimization by nonintimates, childhood abuse, and immigration status, intimate partner violence was associated with an increased probability of developing PTSD. Those women who had experienced intimate partner violence were almost three times more likely to develop PTSD $(95 \% \mathrm{CI}=$ $1.32,6.54)$, which was similar to the result of the simple model described above. No interactions among intimate partner violence (time-varying), childhood abuse, sexual victimization by nonintimates (time-varying), and immigration status were significant.

\section{Discussion}

In this study of a community-based random sample of American women of Japanese descent, the sample proportion estimate of lifetime PTSD was $14 \%$. This proportion is comparable to the $10-14 \%$ lifetime PTSD rates estimated using the sample proportion found in other community-based studies of American women (Breslau et al., 1997; Breslau \& Davis, 1992; Kessler et al., 1995; Resnick et al., 1993), suggesting that rates amorg Japanese American women are similar to those in other sectors of American society. However, as argued in the text, this method of estimation is biased. The Kaplan-Meier estimate of the cumulative probability of PTSD onset by age 49 (the maximum age of the respondents) was $28 \%$, which is about double the sample proportion. The difference represents the consequence of taking censoring into account. Nevertheless, the Kaplan-Meier estimate is probably still an underestimate of lifetime PTSD, as women over the age of 49 may develop PTSD, but were not represented in our sample.

In samples of women from shelters and counseling services, the proportion who currently have a diagnosis of PTSD provides an estimate the prevalence of PTSD in the community (as opposed to lifetime prevalence). This quantity is useful for service providers and helps guide resource allocation, for instance, in estimating the number of women needing mental health services. However, to identify groups of women at risk of developing PTSD and to develop prevention programs, information on the incidence or risk of onset of PTSD over the women's lifecourse is necessary (Greenland \& Rothman, 1998).

Our analyses revealed that the risk of developing PTSD is not constant across a woman's lifetime. The risk of developing PTSD was high during the respondents' mid-20s, dropped to a low in the early 30 s, then started to rise again during the $40 \mathrm{~s}$. Although we might expect younger women to be at increased risk of PTSD because many women had experienced intimate partner violence in their teen years and early 20 s, the increase later in life is rather surprising. Of the five women who developed PTSD during their 40 s, two reported recent intimate partner violence. The remaining three women may have experienced a delayed onset of PTSD, or developed PTSD in response to another type of trauma. Also plausible is that respondents in their late 30s and 40s had experienced PTSD earlier in life, but had difficulty recalling symptoms from their remote past. A study of the same sample of women investigated in this paper (Yoshihama \& Gillespie, 2002) suggests that middle-aged women are less likely than younger women to report intimate partner violence experienced in their youth, and the same phenomenon may occur with PTSD.

Our analyses found no significant interaction between intimate partner violence and time. Thus, there is no evidence that the effect of intimate partner violence decreases over time. However, the lack of significance could be due to the relatively small sample size, and the issue of the duration of the effect of intimate partner violence on PTSD must await further study.

There are several possible limitations of this study. PTSD could have been caused by traumatic events other than intimate partner violence. This study used the National Women's Study PTSD Module and assessed whether respondents had experienced posttraumatic stress symptoms without requiring the respondent to link their symptoms to a specific traumatic event, unlike most other measures of PTSD that assess symptoms in relation to specific event(s). For example, the Composite International Diagnostic Interview (CIDI; World Health Organization, 1997) and SI-PTSD (Davidson, Smith, \& Kudler, 1989) assess symptoms in relation to the most upsetting event, whereas the Diagnostic Interview Schedule (DIS; Robins, Helzer, Croughan, \& Ratcliff, 1981) assesses symptoms in relation to the first, last, and worst events. Recently, Breslau et al. (1998) assessed PTSD in relation to a randomly selected traumatic event from the list of events 
reported by the respondent. Although these approaches would have helped establish the link between intimate partner violence and PTSD, there are limitations. Because many individuals experience multiple traumas, failure to meet diagnostic criteria for selected events does not eliminate the possibility that the respondent has developed PTSD in response to other events. Moreover, the onset of PTSD in relation to an identified event is not necessarily the very first episode of PTSD for that individual; she may have developed PTSD previously in response to another traumatic event. Above all, requiring the respondents to link their symptoms to a specific event can lead to underdetection of PTSD cases because respondents may not be able to link symptoms to a specific event in the past (for more discussion on measurement of PTSD, see Briere, 1997; Solomon, Keane, Newman, \& Kaloupek, 1996). A further limitation of this study is the lack of timing information on trauma other than intimate partner violence and sexual victimization by nonintimates. Thus, we are unable to adjust adequately for other types of trauma in the final model. Although this lack is unfortunate, our data represent an improvement over most studies in which no information on timing is collected, either on the dependent or independent variables.

By collecting information on the timing of intimate partner violence and PTSD, we were able to establish the temporal sequence of these events. PTSD preceded intimate partner violence in four respondents. We included them in the chi-square and Cox time-fixed analysis as this would have been done if no information on timing had been collected. Because we are predicting PTSD onset from intimate partner violence, these respondents inaccurately inflate the relationship between PTSD and intimate partner violence in the chi-square and Cox with time-fixed analysis. However, they do not contribute to the estimate of the relationship between PTSD and intimate partner violence in Cox analysis with time-varying covariates.

Both chi-square analysis and Cox regression with time-fixed covariates found only a marginally significant relationship between intimate partner violence and PTSD. Previous studies have consistently found a significant positive relationship between intimate partner violence and PTSD (Astin, Ogland-Hand, Coleman, \& Foy, 1995; Cascardi et al., 1995; Gleason, 1993; Kemp, Green, Hovanitz, \& Rawlings, 1995). The majority of these studies used a sample of help-seeking battered women who on average had experienced severe intimate partner violence. Compared to help-seeking samples, women in a community-based sample on average experience less severe intimate partner violence; for example, Straus (1990a) found that only $2 \%$ of a national representative sample of women had experienced the severity of abuse equivalent to that experienced by women in shelters. In previous studies (Astin, Lawrence, \& Foy, 1993; Cascardi, O'Leary, \& Schlee, 1999; Houskamp \& Foy, 1991; Kemp et al., 1995; Kemp, Rawlings, \& Green, 1991), a doseresponse relationship has been found between the severity of intimate partner violence and the level of posttraumatic stress symptoms or the probability of developing PTSD. The lack of a significant finding in our study using these methods may be a reflection of a small sample size and the use of a community-based sample.

In contrast, the result from Cox regression with a time-varying covariate was highly significant, even after controlling for other types of trauma. Like other methods of survival analysis, this method accounts for censoring and provides estimates of the changing incidence of PTSD that reflect the changing rates of PTSD over the lifecourse as well as the changing number of individuals at risk. A salient feature of Cox regression with time-varying covariates is its ability to account for the temporal sequence of events. By correctly assessing whether women with PTSD had experienced intimate partner violence in the past, this method showed that the relationship between intimate partner violence and PTSD was strong and highly significant. Future studies on the relationship between intimate partner violence and PTSD should collect information on the timing of intimate partner violence and the onset of PTSD. This would allow the use of this powerful statistical technique.

\section{Acknowledgments}

We thank Brenda Gillespie for her expertise and Terri Torkko for her assistance in preparation of this manuscript. Data collection for this study was supported by a grant from the National Institute of Mental Health (RO3 MH54351-01), and data analysis and manuscript preparation were supported in part by a grant from the National Institute of Mental Health (5 R24 MH51363 5).

\section{References}

Abueg, F. R., \& Chun, K. M. (1996). Traumatization stress among Asians and Asian Americans. In A. J. Marsella, M. J. Friedman, E. T. Gerrity, \& R. M. Scurfield (Eds.), Ethnocultural aspects of posttraumatic stress disorder: Issues, research, and clinical applications (pp. 285-299). Washington, DC: American Psychological Association.

Allensworth, E. M. (1997). Earnings mobility of first and "1.5" generation Mexican-origin women and men: A comparison with US-born Mexican Americans and non-Hispanic Whites. International Migration Review, 31, 386-410.

Allison, P. (1995). Survival analysis using the SAS System: A practical guide. Cary, NC: SAS Institute. 
American Psychiatric Association. (1994). Diagnostic and statistical manual of mental disorders (4th ed.). Washington, DC: Author.

Astin, M. C., Lawrence, K. J., \& Foy, D. W. (1993). Posttraumatic stress disorder among battered women: Risk and resiliency factors. Violence and Victims, 8, 17-28.

Astin, M. C., Ogland-Hand, S. M., Coleman, E. M., \& Foy, D. W. (1995). Posttraumatic stress disorder and childhood abuse in battered women: Comparisons with maritally distressed women. Journal of Consulting and Clinical Psychology, 6, 308-312.

Breslau, N., \& Davis, G. C. (1992). Posttraumatic stress disorder in an urban population of young adults: Risk factors for chronicity. American Journal of Psychiatry, 149, 671-675.

Breslau, N., Davis, G. C., Peterson, E. L., \& Schultz, L. (1997). Psychiatric sequelae of posttraumatic stress disorder in women. Archives of General Psychiatry, 54, 81-87.

Breslau, N., Kessler, R. C., Chilcoat, H. D., Schultz, L. R., Davis, G. C., \& Andreski, P. (1998). Trauma and posttraumatic stress disorder in the community: The 1996 Detroit Area Survey of Trauma. Archives of General Psychiatry, 55, 626-632.

Briere, J. (1997). Trauma-specific self-report measures. In J. Briere (Ed.), Psychological assessment of adult posttraumatic states (pp. 127146). Washington, DC: American Psychological Association.

Cascardi, M., O'Leary, K. D., Lawrence, E. E., \& Schlee, K. A. (1995). Characteristics of women physically abused by their spouses and who seek treatment regarding marital conflict. Journal of Consulting and Clinical Psychology, 63, 616-623.

Cascardi, M., O'Leary, K. D., \& Schlee, K. A. (1999). Co-occurrence and correlates of posttraumatic stress disorder and major depression in physically abused women. Journal of Family Violence, 14, 227 249.

Chilcoat, H. D., \& Breslau, N. (1998). Investigations of causal pathways between PTSD and drug use disorders. Addictive Behaviors, 23, $827-840$.

Davidson, J., Smith, R., \& Kudler, H. (1989). Validity and reliability of the DSM-III criteria for posttraumatic stress disorder: Experience with a structured interview. Joumal of Nervous and Mental Disease, $177,336-341$.

Gleason, W. J. (1993). Mental disorders in battered women: An empirical study. Violence and Victims, 8, 53-68.

Greenland, S., \& Rothman, K. J. (1998). Modern epidemiology. Philadelphia: Lippincott-Raven.

Houskamp, B. M., \& Foy, D. W. (1991). The assessment of posttraumatic stress disorder in battered women. Joumal of Interpersonal Violence, 6, 367-375.

Kaplan, E. L., \& Meier, P. (1958). Nonparametric estimation from incomplete observations. Joumal of the American Statistical Association, $53,457-481$.

Kemp, A., Green, B., Hovanitz, C., \& Rawlings, E. (1995). Incidence and correlates of posttraumatic stress disorder in battered women: Shelter and community samples. Journal of Interpersonal Violence, $10,43-55$.

Kemp, A., Rawlings, E. I., \& Green, B. L. (1991). Post-traumatic stress disorder (PTSD) in battered women: A shelter sample. Journal of Traumatic Stress, 4, 137-148.

Kessler, R. C., Sonnega, A., Bromet, E., Hughes, M., \& Nelson, C. B. (1995). Postraumatic-stress-disorder in the National Comorbidity Survey. Archives of General Psychiatry, 52, 1048-1060.

Kilpatrick, D. G., Resnick, H. S., Saunders, B. E., \& Best, C. L. (1989). National Women's Study PTSD module. Unpublished instrument by the National Crime Victims Research and Treatment Center, Charleston, SC.
Kim-Hog, M., Chong, S., Blake, D. D., \& Hiley-Young, B. (1995). Psychological impact of the Los Angeles riots on Korean-American victims: Implications for treatment. American Journal of Orthopsychiatry, 65, 138-146.

Koss, M., Gidycz, C., \& Wisniewski, N. (1987). The scope of rape: Incidence and prevalence of sexual aggression and victimization in a national sample of higher education students. Journal of Consulting and Clinical Psychology, 255, 162-170.

Loo, C. M. (1994). Race-related PTSD: The Asian American Vietnam veteran. Joumal of Traumatic Stress, 7, 637-656.

Marin, G., \& Marin, B. V. (1991). Reseanch with Hispanic populations. Newbury Park, CA: Sage.

Myers, D., \& Cranford, C. J. (1998). Temporal differentiation in the occupational mobility of immigrant and native-bom Latina workers. American Sociological Review, 63, 68-93.

Resnick, H. S., Kilpatrick, D. G., Dansky, B. S., Saunders, B. E., \& Best, C. L. (1993). Prevalence of civilian trauma and posttraumatic stress disorder in a representative national sample of women. Journal of Consulting and Clinical Psychology, 61, 984-991.

Robins, L. N., Helzer, J. E., Croughan, J. L., \& Ratcliff, K. S. (1981). National Institute of Mental Health diagnostic interview schedule: Its history, characteristics, and validity. Archives of General Psychiatry, 38, 381-389.

Russell, D. E., \& Howell, N. (1983). The prevalence of rape in the United States revised. Signs, 8, 688-695.

Smith, M. D. (1987). The incidence and prevalence of women abuse in Toronto. Violence and Victims, 2, 173-187.

Solomon, S. D., Keane, T. M., Newman, E., \& Kaloupek, D. G. (1996). Choosing self-report measures and structured interviews. In E. B. Carison (Ed.), Trauma research methodology (pp. 57-81). Lutherville, MD: Sidran Press.

Straus, M. A. (1990a). Injury and frequency of assault and the "representative sample fallacy" in measuring wife beating and child abuse. In M. A. Straus \& R. J. Gelles (Eds.), Physical violence in American families: Risk factors and adaptations to violence in 8,145 families (pp. 75-91). New Brunswick, NJ: Transaction Publications.

Straus, M. A. (1990b). Measuring intrafamily conflict and violence: The Conflict Tactics (CT) Scale. In M. A. Straus \& R. J. Gelles (Eds.), Physical violence in American families: Risk factors and adaptations to violence in 8,145 families (pp. 29-47). New Brunswick, NJ: Transaction Publications.

Therneau, M. T., \& Grambsch, P. M. (2000). Modeling survival data. Extending the Cox model. New York: Springer.

Tolman, R. M., \& Rosen, D. (2001). Domestic violence in the lives of women receiving welfare: Mental health, substance dependence and economic well-being. Violence Against Women, 7, 141-158.

World Health Organization. (1997). Composite International Diagnostic Interview ( $C I D I$ ): Core version 2.1. Geneva, Switzerland: Author.

Wyatt, G. E. (1985). The sexual abuse of Afro-American and White American women in childhood. Child Abuse and Neglect, 9, 507519.

Yoshihama, M. (1999). Domestic violence against women of Japanese descent in Los Angeles: Two methods of estimating prevalence. Violence Against Women, 5, 869-897.

Yoshihama, M., \& Gillespie, B. (2002). Age adjustment and recall bias in the analysis of domestic violence data: Methodological improvement through the application of survival analysis methods. Journal of Family Violence, 17, 199-221.

Yoshihama, M., \& Horrocks, J. (2002). Posttraumatic stress symptoms and victimization among Japanese American women. Journal of Consulting and Clinical Psychology, 70, 205-215. 